\title{
Could interferon help treat Ebola?
}

I $\mathrm{n}$ early August, amid rising alarm about Ebola, immunologist Eleanor Fish of the University Health Network in Toronto contacted a friend in Sierra Leone to say that the antiviral drug interferon might be helpful. There are no approved treatments for Ebola, but Fish, an expert on interferon, knew of a study published last year that showed interferon prolongs survival in monkeys infected with Ebola and the related Marburg virus.

She heard back from an assistant to Sierra Leone President Ernest Bai Koroma and then from a World Health Organization (WHO) official, who asked her to join a group of scientists charged with developing a list of potential Ebola therapies and vaccines. After two weeks of discussions, the group headed to WHO headquarters in Geneva to make their case at an international research consultation, but once there, Fish says, they got a reality check from "the African communities that were affected, in terms of their urgency in wanting some action."

Very few of the interventions the group considered can meet that urgent demand. The experimental drug ZMapp, developed in part at the Public Health Agency of Canada, has been used by seven people infected with Ebola. But it will be out of stock for months and is given intravenously, which is a challenge in Ebola-affected areas.

Another intravenous drug, TKMEbola, being developed by the British Columbia company Tekmira Pharmaceuticals Corp. with funding from the US Department of Defense, has a special exemption to be used for individu-

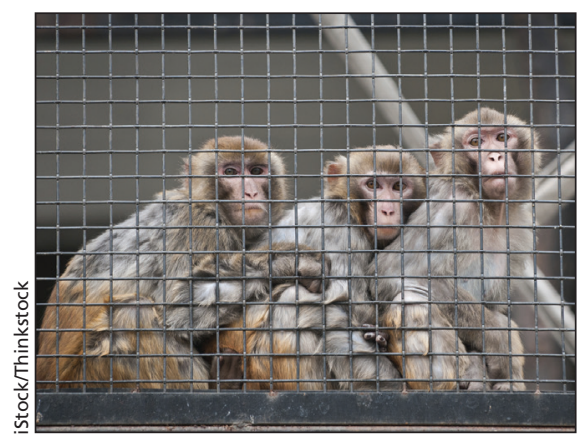

Among medication options shown to prolong survival in Ebola-infected monkeys, only interferon is commercially available and has a safety profile for use in humans. als infected with Ebola, though it's in a Phase-1 human safety trial.

Among treatment options, only interferon is commercially available and, says Fish, it's the only one with a "safety profile in humans." The drug is approved by the US Food and Drug Administration.

David Kelvin, a virus expert at the Toronto General Research Institute, cautions that the safety profile may not apply, "because you can give a drug for toxicity to a healthy person and it's very different from giving it to somebody who actually is infected with a virus." But Kelvin believes a "credible study" of interferon should go forward.

Dr. Michael Kurilla, director of the Office of Biodefense Research Affairs at the US National Institutes of Health (NIH), says that while it's not a cure, it does have a role.

"If you can get interferon in early on, in the nonhuman primate model, what you see is a delay to death and a reduction in the level of viremia, both of which would seem to be good."

The NIH is discussing a combination therapy - adding interferon to another available antiviral drug, ribavirin - and studying the combination in monkeys. If it proves to be efficacious, it "might be something easily implementable in patients," Kurilla says.

WHO Assistant Director-General Dr. Marie-Paule Kieny says interferon could be tried in patients "with early disease."

But the Liberian and Guinean delegations in Geneva aren't waiting for the WHO's opinion. They've already asked Fish about the drug and she has written a protocol that they can share with local clinicians. The drug she suggests using, Infergen, is made in Germany and distributed by Ukraine-based Pharmunion BSV development, which will donate it in the amounts needed.

They may begin by trying the drug on 20 patients at a centre in Conakry, Guinea's capital. "Maybe that's the start," says Fish, "and based on that we can very rapidly roll out elsewhere." Miriam Shuchman, Toronto, Ont.

CMAJ 2014. DOI:10.1503/cmaj.109-4906 